\title{
End-to-End Delay Bound for VR Services in 6G Terahertz Networks with Heterogeneous Traffic and Different Scheduling Policies
}

\author{
Benedetta Picano (D)
}

check for updates

Citation: Picano, B. End-to-End Delay Bound for VR Services in 6G Terahertz Networks with Heterogeneous Traffic and Different Scheduling Policies. Mathematics 2021, 9, 1638. https://doi.org/10.3390/ math9141638

Academic Editor: Daniel-Ioan Curiac

Received: 3 June 2021

Accepted: 9 July 2021

Published: 12 July 2021

Publisher's Note: MDPI stays neutral with regard to jurisdictional claims in published maps and institutional affiliations.

Copyright: (C) 2021 by the author. Licensee MDPI, Basel, Switzerland. This article is an open access article distributed under the terms and conditions of the Creative Commons Attribution (CC BY) license (https:// creativecommons.org/licenses/by/ $4.0 /)$.
Department of Information Engineering, University of Florence, 50139 Firenze, Italy; benedetta.picano@unifi.it

\begin{abstract}
The emerging sixth-generation networks have to provide effective support to a wide plethora of novel disruptive heterogeneous applications. This paper models the probabilistic endto-end delay bound for the virtual reality services in the presence of heterogeneous traffic flows by resorting to the stochastic network calculus principles and exploiting the martingale envelopes. The paper presents the network performance analysis under the assumption of different scheduling policies, considering both the earliest deadline first and the first-in-first-out queue discipline. Furthermore, differently from previous literature, the probabilistic per-flow bounds have been formulated taking into account a number of traffic flows greater than two, which results in a theoretical analysis that is remarkably more complex than the case in which only two concurrent flows are considered. Finally, the validity of the theoretical bounds have been confirmed by the evident closeness between the analytical predictions and the actual simulation results considering, for the sake of argument, four concurrent traffic flows with heterogeneous quality-of-service constraints. That closeness exhibits the ability of the proposed analysis in fitting the actual behavior of the system, representing a suitable theoretical tool to support resource allocation strategies, without violating service constraints.
\end{abstract}

Keywords: heterogeneous traffic; terahertz communications; stochastic network calculus

\section{Introduction}

The ultimate virtual reality $(\mathrm{uVR})$ services are one of the most anticipated applications of the new era sixth generation (6G) networks. Hallmarks of the uVR services are the stringent quality of service (QoS) requirements, as yet imposed by the more general class of the virtual reality (VR) services to which they belong, and the fact that $\mathrm{uVR}$ applications solicit all the five senses [1-3]. The $\mathrm{uVR}$ services require communications characterized by both high rate and low latency (HRLLC) constraints. Due to the strict delay requirements imposed by the $\mathrm{uVR}$ services, the usage of the high frequencies bands, such as $30-300 \mathrm{GHz}$ band millimeter waves (mmWaves) and free-space optical in the range of 200-385 THz [4], represent a natural candidate to guarantee short-range HRLLC.

Generally speaking, the uVR services are supported by a dedicated hardware, that is, the VR equipment (VE), typically head-mounted displays, needed for a stereoscopic visual experience, images rendering, and so on [5,6], which aims to boost the immersive experience of the uVR applications offered to the users. Furthermore, one of the major concerns regarding the feasibility of the practical realization of the $\mathrm{uVR}$ services lies in the inability of today's VEs in supporting local rendering, observing the QoS imposed by the high-flying uVR applications, since they are not powerful enough. Indeed, the primary limit of the VEs is represented by the battery lifetime, whose moderate capability does not provide proper support to prolonged rendering sessions, triggering excessive heat and battery degradation [5,7]. In this circumstance, the pervasive edge computing (PEC) paradigm has emerged as a promising candidate to offer support to wireless uVR environments $[5,7,8]$, hosting the tasks rendering computations, offloaded by the users' 
devices towards computing-assisted edge nodes (e.g., small base stations), in order to limit the energy consumption at the VE's site and to preserve the battery lifetime [7].

The 6G PEC network may represent a valuable environment for handling $\mathrm{uVR}$ services. Nevertheless, the next generation applications give rise to a novel network concept, in which the presence of heterogeneous traffic demand is a point needing further and accurate investigation. For this reason, the analysis of the system's performance in guaranteeing the QoS required by the $\mathrm{uVR}$ services in the presence of concurrent heterogeneous traffic flows is crucial, allowing improvement of the proper system's exploitation.

In fact, the main goal of the paper is the investigation of the potentialities of a $6 \mathrm{G}$ PEC network in handling the $\mathrm{uVR}$ traffic flow when it coexists with other flows and is heterogeneous in QoS constraints. The analysis has been provided in terms of endto-end (e2e) application delay, formulating a per-flow e2e probabilistic bound under the assumption of both the earlier deadline first (EDF) and the first-in-first-out (FIFO) scheduling policies. The e2e delay bounds have been modeled by resorting to the stochastic network calculus (SNC), a credited theoretical framework particularly suitable for studying computer networks' behavior in terms of delay [9-11]. Throughout the application of the min-plus algebra principles, the SNC allows the formulation of probabilistic bounds for the networks, providing flexibility in tackling cascade network systems, in which the service curve of the e2e system is represented by the convolution of the service curves of each individual subsystem [12].

This paper investigates the per-flow e2e delay performance analysis of $\mathrm{uVR}$ services within a tandem 6G PEC network operating at THz frequencies, exploiting both the SNC and the martingale theory fundamentals [13], Consequently, the contributions of this paper, in comparison to the recent literature, are constituted by:

- The system performance analysis of a 6G PEC network's behavior, operating at terahertz frequencies, in supporting the $\mathrm{uVR}$ services. The whole scenario has been modeled as a tandem system, in which the first subsystem represents the access towards the PEC node in order to send the packet needing computation, the second subsystem is the computation module, and the last subsystem models the transmission of the packet back to the user. The access subsystem is without contention, the computation module is shared among different traffic flows heterogeneous in QoS, which compete with each other to receive the service. Then, the tasks pass through the transmission subsystem to be sent back to the device;

- Formulation of the per-flow e2e delay bound of services in the presence of concurrent traffic flows, under the assumption of both the EDF and the FIFO scheduling policies. The proposed per-flow bounds have been modeled by resorting to the application of the SNC principles together with the martingale envelopes, which are a recognized theoretical tool capable of empowering the analytical prediction about the network's behavior;

- The validation of the proposed analytical bounds that exhibit remarkable closeness between the theoretical results and the actual simulation outcomes, for both the considered scheduling policies. In fact, the per-flow e2e delay bounds achieve accurate performance predictions about the reliability of the PEC network in guaranteeing the QoS constraints imposed by the heterogeneous traffic flows. It is important to highlight that, due to the tightness of the proposed bound to the simulation results, the analytical prediction formulated is particularly suitable in the planning design phase of a next generation network that aims to guarantee fixed QoS requirements, providing the chance to perform proper resource allocation strategies.

Furthermore, it is important to highlight here that the proposed performance bounds are useful for predicting the behavior of the network object of the analysis, without resorting to the numerical simulations. In fact, as detailed in the Performance Analysis section, the bounds formulated present a remarkable accuracy in fitting the actual network behavior, under the assumption of different scheduling policies. Such a tightness between the theoretical bounds and the simulation results enables the application of the proposed 
analytical bounds as a predictive tool during the network management process, representing a powerful method for forecasting the network reliability under different network loads and resources hypotheses, avoiding the extensive and time-consuming network testing for each case considered. In fact, the proposed analytical predictions may be functionally integrated within more general network optimization frameworks, in which the extensive long-term network simulations are not an option.

The rest of the paper is organized as follows: Section 2 proposes an in-depth review of the prior works related to the problem addressed here. Section 3 details the system model, and in Section 4 the SNC and the martingale theory fundamentals are briefly recalled. Then, the e2e delay bounds formulation is presented in Section 4, while in Section 6 the performance analysis is discussed. Finally, our conclusions are drawn in Section 7.

\section{Related Works}

Both the ultra-reliable low latency and the enhanced mobile broadband communications have been deeply investigated in [14], where the stochastic geometry was applied. The joint optimization of the caching and computation offloading problems has been presented in [15], defining proper management policies to minimize the mean transmission rate, on the basis of the quality of service constraints imposed by the VR application services. Furthermore, in [16] the problem of the joint satisfaction of both the reliability and low latency requirements is addressed, proposing to design a massive multiple-inputmultiple-output and multi-connectivity access protocol. Differently, the packet error rate in mission critical communications is the main focus of [17], which proposes the development and implementation of a statistical framework for ultra reliable low latency communications systems. Moreover, the framework object of the study is constituted by three modules: the channel model selection, the learning model, and the transmission rate selection. The authors of [18] propose an ad-hoc network simulator for the VR environments [18], where a master-slave architecture has been designed, and a game engine has been exploited as support. The next generation VR devices over wireless local area networks were also the main target in [19], in which the delay due to the wireless channel fluctuations has been deeply analyzed, and a multi-user VR channel access scheme has been proposed, aiming at reducing the network delay, reaching a better performance than the conventional channel access schemes. An e2e delay bound based on the SNC and the martingale theory has been defined in [20], in which the multi-hop delay in the vehicular ad hoc networks is analyzed, emphasizing the analysis of both the access and the queuing delay. The authors of [21] provide the e2e delay analysis of both delay tolerant and delay sensitive bursty flows. In this case, the proposed probabilistic bounds have also been formulated by resorting to the martingale theory. Similarly, the martingale bounds have also been derived in [22], considering the ultra reliable low latency and the massive machine type communications. The main goal of the authors of [22] is the maximization of the throughput, taking into account a multi-channel aloha type grant free scheme. The multi-variable-grey wolf optimizer algorithm has been applied to the problem formulated. Moreover, the martingale envelopes are also used in [13], where the authors propose the analysis of the major random access scheme, in terms of both the packets backlog and delay.

This paper proposes the probabilistic e2e delay bound formulation involving the SNC principles and the martingale theory, considering a multi-hop tandem system. It is important to highlight here that the proposed e2e delay bounds, modeled under different scheduling policies assumptions, are derived considering a number of traffic flows greater than two, which increases the difficulty of the analysis. Furthermore, different to the standard queueing theory [23], the computation service time is not exponentially distributed, proposing a more general connotation to the analysis here provided in comparison to the more conventional Markovian analysis [23]. Finally, to the best of the author's knowledge, this paper is the first that takes into account the access delay and the presence of concurrent heterogeneous traffic with different scheduling policies, within the wireless VR environments. 


\section{System Model}

In this section, the system model will be described, considering four classes of traffic type, in order to provide a concrete example to the proposed probabilistic bound formulated in the next section. In this regard, it is important to clarify that the previous literature models the same bound considering a number of traffic flows equal to two. Differently, this paper extends the proposed analysis to a number of flows, greater than two, which makes the analysis remarkably more complex.

In accordance with Figure 1, the paper considers four classes of traffic: the uVR traffic $(\mathcal{H})$, other human-based traffic, such as online gaming $\mathcal{G}$, and two other machine type traffics, hereafter referred to as $\mathcal{C}$ and $\mathcal{D}$, characterized by less stringent QoS constraints in comparison to the flow $\mathcal{H}$, but with heterogeneous QoS requirements. More in depth, $\mathcal{C}$ and $\mathcal{D}$ denote device communities, which are injected into the network packets resulting from the data collected by a plethora of underlying sensors. All the devices in the network send data packets of equal size and time duration to the same edge computing node, hereafter referred to as the Small Base Station (SBS) or PEC node (PECN) interchangeably, which operates at $\mathrm{THz}$ frequencies. Furthermore, the four traffic flows here considered have a channel dedicated to the PEC. Considering the time as divided into slots of duration equal to the packet transmission time, the slots are assigned to the $\mathrm{uVR}$ user in an exclusive (no-contention) mode, as well as for the traffic $\mathcal{G}$. Differently, the elements of each device community, $\mathcal{C}$ and $\mathcal{D}$, access the channel following the well-known contention based Aloha scheme as detailed later on. Furthermore, the analysis of the machine devices' traffic is performed assuming a saturation condition, that is, each device always has a new packet ready to be transmitted whenever an access attempt is successfully accomplished [24]. According to $[25,26]$, we have assumed that each device is located at a fixed distance $d_{0}$ from the tagged SBS.

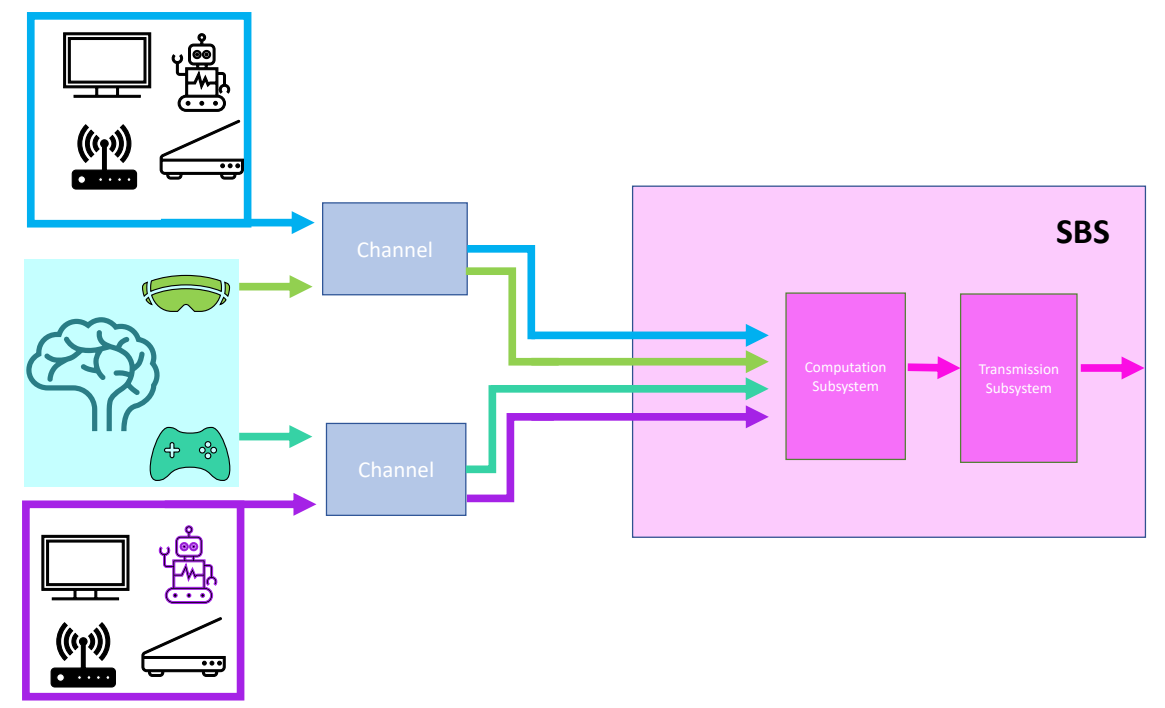

Figure 1. System Scenario.

\subsection{Channel Model}

In reference to [25-27], we have considered a high-dense scenario with short range communications, in which all the devices have the availability of a continuous line-ofsight (LoS) connection with the reference PECN. Therefore, a channel model, as presented in [25-27], has been assumed. Let $\mathcal{R}$ be the instantaneous rate for the uplink/downlink LoS communications between the tagged SBS and the linked device. Consequently, we have $[25,26]$

$$
\mathcal{R}=\mathcal{W} \log _{2}\left(1+\frac{P A_{0} d_{0}^{-2} e^{-K(f) d_{0}}}{N_{0}}\right),
$$


with $\mathcal{W}$ expressing the bandwidth of the communication link, $N_{0}$ considering both the molecular absorption noise and the Johnson-Nyquist noise at the receiving site, and given by

$$
N_{0}=\frac{\mathcal{W} \zeta}{4 \pi} g_{B} T_{0}+P A_{0} d_{0}^{-2}\left(1-e^{-K(f) d_{0}}\right)
$$

in which $g_{B}$ denotes the Boltzmann constant, $T_{0}$ is the temperature in Kelvin, $\zeta$ is the wavelength, $K(f)$ is the global absorption coefficient of the medium and $A_{0}=\frac{c^{2}}{16 \pi^{2} f^{2}}[25,26]$.

\subsection{Channel Access Scheme}

The uVR traffic flow, that is, $\mathcal{H}$, is characterized by strict delay constraints, and the VE, which originates the flow $\mathcal{H}$, accesses the channel towards the PECN on a given slot without any contention, with probability $p_{\mathcal{H}}$. The same access scheme is adopted by the human based traffic $\mathcal{G}$, which is typically slightly less stringent in delay QoS than the traffic $\mathcal{H}$, accessing each slot with probability $p_{\mathcal{G}}$. It is important to note here that both the $\mathcal{H}$ and the $\mathcal{G}$ flows have a dedicated channel. Furthermore, let $v$ be the number of machine devices belonging to $\mathcal{C}$. Within the device community, the $v$ elements access the dedicated channel using the Aloha contention based scheme. Each machine type device manages the transmission of only one packet at a time, having a transmission success when only one packet is transmitted into a slot. However, if two or more packet transmission attempts occur in the same slot, a collision happens, making transmission impossible. Therefore, a new packet can be transmitted to a given slot with $p_{c}$, as with any other packet involved in collisions until a success occurs. Finally, for the sake of analysis tractability, we assumed that the LoS link cannot be lost once a transmission was started. It is important to highlight here that the same assumptions have been made for the machine type devices belonging to $\mathcal{D}$, whose access probability is given by $p_{d}$. Finally, without loss of generality, the number of devices belonging to $\mathcal{D}$ has been assumed to be equal to $v$.

\section{Stochastic Network Calculus Principles}

Stochastic Network Calculus Fundamentals

In this section, the fundamentals of the SNC are recalled, with reference to Figure 2.

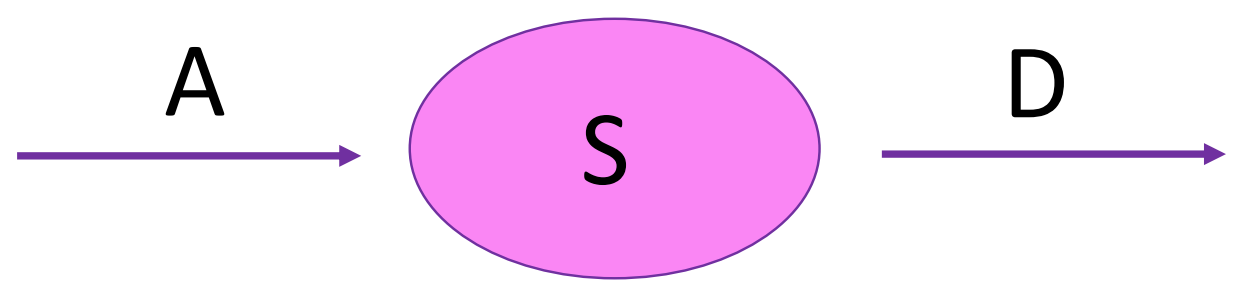

Figure 2. Single server scenario. A: arrival process, S: service process, D: departure process.

Let $T=[a, b]$ be the time interval during which the system illustrated in Figure 2 is observed. Therefore, the cumulative number of arrivals at the server node during $T$ is given by [28]

$$
A_{a, b}=A(a, b)=\sum_{t=a+1}^{b} X_{t}
$$

in which $X_{t}$ is the number of packets arriving at time $t$.

Likewise, $S_{a, b}=S(a, b)=\sum_{t=a+1}^{b} S_{t}$ is the bivariate service process.

Arrival and service processes have been assumed to be stationary, ergodic, statistically independent and reversible, in compliance with the standard literature [13]. These assumptions turn out to be reasonable due to the type of access scheme adopted. In fact, it is compliant with the models proposed in papers $[13,20]$, which investigate the feasibility of these hypotheses. However, it is important to note here that the in-depth discussion of these aspects is out of the scope of this paper. Referring to $\otimes$ as the $(\min ,+)$ convolution 
operator, considering $A(g)=A(0, g)$, the bivariate departure process $D(a, b)$ is defined as follows:

$$
D(b)=D(0, b) \geq A \otimes S(0, b):=\inf _{0 \leq g \leq b}\{A(g)+S(g, b)\} .
$$

Then, as in $[9,20,28]$, the delay process $W(b)$ is given by

$$
W(b)=W(0, b)=\inf \{c \geq 0 \mid A(b-c) \leq D(b)\},
$$

from which it follows that

$$
W(b) \geq c \text { iff } A(b-c) \geq D(b) .
$$

As a consequence, in accordance with [28], the complementary cumulative distribution as a function of $W(b)$ can be defined as:

$$
\mathbb{P}(W(b)>c)=\mathbb{P}(A(b-c) \geq D(b))
$$

It is important to highlight that, according to the standard SNC theory, the bounds result in being characterized by the arrival processes only. By applying the martingale envelopes combined with the SNC, whose main definitions are reported in Appendix A, a proper exponential transformation can be formulated, in which both the arrivals and service processes are taken into account.

\section{End-to-End Delay Analysis}

This section provides the per-flow e2e delay analysis considering the system depicted in Figure 3.

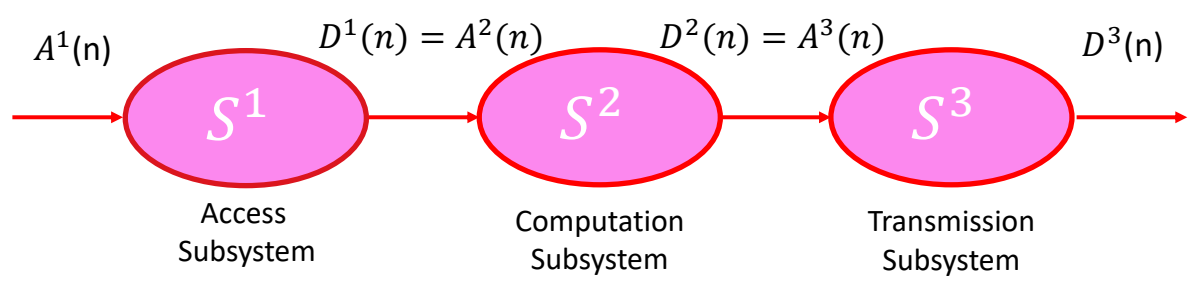

Figure 3. Tandem network.

Let $A$ be the aggregated arrivals at the central unit. Therefore, $A$ is clearly expressible as $A=\sum_{t=0}^{T} X_{t}$, with $X_{t}$ given by (8), in which

$$
\begin{aligned}
& \left(\begin{array}{ll}
4, & p_{\mathcal{H}} p_{\mathcal{G}} p_{\mathcal{C}} p_{\mathcal{D}} \\
3, & p_{\mathcal{G}} p_{\mathcal{H}} p_{\mathcal{C}}\left(1-p_{\mathcal{D}}\right)+p_{\mathcal{G}} p_{\mathcal{H}} p_{\mathcal{D}}\left(1-p_{\mathcal{C}}\right)+p_{\mathcal{C}} p_{\mathcal{D}} p_{\mathcal{H}}\left(1-p_{\mathcal{G}}\right)
\end{array}\right. \\
& +p_{\mathcal{C}} p_{\mathcal{D}} p_{\mathcal{G}}\left(1-p_{\mathcal{H}}\right) \\
& \begin{array}{l}
2, \quad p_{\mathcal{G}} p_{\mathcal{H}}\left(1-p_{\mathcal{C}}\right)\left(1-p_{\mathcal{D}}\right)+p_{\mathcal{G}} p_{\mathcal{C}}\left(1-p_{\mathcal{H}}\right)\left(1-p_{\mathcal{D}}\right)+p_{\mathcal{G}} p_{\mathcal{D}}\left(1-p_{\mathcal{C}}\right)\left(1-p_{\mathcal{H}}\right) \\
\quad+p_{\mathcal{H}} p_{\mathcal{C}}\left(1-p_{\mathcal{G}}\right)\left(1-p_{\mathcal{D}}\right)+p_{\mathcal{H}} p_{\mathcal{D}}\left(1-p_{\mathcal{G}}\right)\left(1-p_{\mathcal{C}}\right)
\end{array} \\
& +p_{\mathcal{C}} p_{\mathcal{D}}\left(1-p_{\mathcal{H}}\right)\left(1-p_{\mathcal{G}}\right) \\
& 1, \quad p_{\mathcal{G}}\left(1-p_{\mathcal{H}}\right)\left(1-p_{\mathcal{C}}\right)\left(1-p_{\mathcal{D}}\right)+p_{\mathcal{H}}\left(1-p_{\mathcal{G}}\right)\left(1-p_{\mathcal{C}}\right)\left(1-p_{\mathcal{D}}\right) \\
& +p_{\mathcal{C}}\left(1-p_{\mathcal{G}}\right)\left(1-p_{\mathcal{H}}\right)\left(1-p_{\mathcal{D}}\right)+p_{\mathcal{D}}\left(1-p_{\mathcal{G}}\right)\left(1-p_{\mathcal{C}}\right)\left(1-p_{\mathcal{H}}\right) \\
& 0, \quad\left(1-p_{\mathcal{G}}\right)\left(1-p_{\mathcal{H}}\right)\left(1-p_{\mathcal{C}}\right)\left(1-p_{\mathcal{D}}\right) \text {. }
\end{aligned}
$$




\subsection{Martingale Bound for FIFO Policy}

Under the assumption of the FIFO scheduling discipline, the packets are served in accordance with their arrival time. Let $A^{-i}$ be the cumulative arrivals of the flows except the $i$-th flow. Therefore, as detailed in [29], the FIFO scheduling for the generic type flow $i$, $i \in\{\mathcal{H}, \mathcal{G}, \mathcal{C}, \mathcal{D}\}$ is defined as:

$$
S^{i}=\left[S^{T o t}(m, n)-A^{-i}(m, n-x)\right]_{+} \mathbf{1}_{n-m>x},
$$

where $S^{T o t}$ is the service curve of the network, obtained via the min-plus convolution of the service curves of each server, $x$ is a fixed parameter freely chosen, and $\mathbf{1}$ is the indicator function assuming value 1 if the condition $n-m>x$ is satisfied, and zero otherwise, according to $[9,12,28,29]$. Assuming that the arrivals flows and the services admit the martingale envelopes, hereafter referred to as $M_{A^{i}}, i \in\{\mathcal{H}, \mathcal{G}, \mathcal{C}, \mathcal{D}\}$ and $M_{S^{i}} i \in\{2,3\}$, respectively, we can affirm that $[9,12,28]$

$$
D(n) \geq \inf _{0 \leq k \leq n}\left\{A^{i}(l)+S^{2} \otimes S^{3}(l, n)\right\},
$$

from which it follows that

$$
D(n) \geq A^{i} \otimes S^{2} \otimes S^{3}(l, n) .
$$

Similarly, from (5)-(7), for the generic flow $i$, we have

$$
\mathbb{P}(W(n) \geq k) \leq \mathbb{P}\left(\sup _{0 \leq k \leq n}\left\{A^{i}(k, n)-S^{i}(n)\right\} \geq 0\right),
$$

from which we obtain

$$
\mathbb{P}(W(n) \geq k) \leq \mathbb{P}\left(\sup _{0 \leq k \leq n}\left\{A^{i}(k, n)-\left[S^{T o t}(n)-A^{-i}(n)\right]\right\} \geq 0\right) .
$$

Therefore, by applying the relation $S^{T o t}=S^{1} \otimes S^{2}$ to (13), we have:

$$
\begin{aligned}
& \mathbb{P}(W(n) \geq k) \leq \\
& \mathbb{P}\left(\sup _{0 \leq k \leq n}\left\{A^{i}(k, n)+A^{-i}(n)-S^{2}(n) \otimes S^{3}(n)\right\} \geq 0\right) .
\end{aligned}
$$

Hence, when $\tau_{1}+\tau_{2}=n$, without loss of generality we can assume the services are homogeneous and independent. Consequently, $K^{S^{2}}=K^{S^{3}}=K_{s}$. Then, we introduce the envelopes on the basis of the supermartingale approximations as

$$
\begin{gathered}
M_{A^{i}} \approx h_{A^{i}}\left(a_{n}^{i}\right) e^{\theta\left(A^{i}(k, n)-(n-k) K^{A^{i}}\right)}, \quad i \in\{\mathcal{H}, \mathcal{G}, \mathcal{C}, \mathcal{D}\}, \\
M_{S^{j}} \approx h_{S^{j}}\left(s_{\tau_{j}}\right) e^{\theta\left(\tau_{i} K_{s}-S^{j}\left(\tau_{j}\right)\right)} .
\end{gathered}
$$

As a consequence of the independence assumption, the supermartingale process detailed in (17) is built. The key point here is that, applying the martingale properties [30], the product of the supermartingale processes is a supermartingale process. Therefore, the stopping theorem [30] can be applied, and the supermartingale process results, stemming from the product of Equations (15) and (16), given by:

$$
\mathcal{M}=\prod_{j \in\left\{A^{\mathcal{H}}, A^{\mathcal{G}}, A^{\mathcal{C}}, A^{\mathcal{D}}, S^{2}, S^{3}\right\}} M_{j} .
$$


At this point, by invoking the property that the expectation of a supermartingale is non-decreasing and the independent assumption as in [13], we have that

$$
\mathbb{E}[\mathcal{M}(k)]=\mathbb{E}\left[\prod_{i \in\{\mathcal{H}, \mathcal{G}, \mathcal{C}, \mathcal{D}\}} M_{A^{i}}(0) \prod_{j=2}^{3} M_{S^{j}}\right] .
$$

As a consequence of (18), applying the optional stopping theorem [31], we have:

$$
\mathbb{E}[\mathcal{M}(k)] \leq \prod_{i \in\{\mathcal{H}, \mathcal{G}, \mathcal{C}, \mathcal{D}\}} \mathbb{E}\left[M_{A^{i}}(0)\right] \prod_{j=2}^{3} \mathbb{E}\left[M_{S^{j}}(0)\right] .
$$

Then, from (12) and (13), the bound results are

$$
\mathbb{P}(W(n) \geq k) \leq e^{-\theta^{*} k K_{s}} B,
$$

where

$$
B=\frac{\left[\prod_{i \in\{\mathcal{H}, \mathcal{G}, \mathcal{C}, \mathcal{D}\}} \mathbb{E}\left[M_{A^{i}}(0)\right]\right] \mathbb{E}\left[M_{S^{2}}(0)\right] \mathbb{E}\left[M_{S^{3}}(0)\right]}{H},
$$

where $H=\min \left\{h_{A^{1}}\left(a_{n}^{1}\right) h_{S^{i}}\left(s_{\tau_{i}}\right): a_{n}-s_{\tau_{i}}>0\right\}$, and $\theta^{*}=\sup \left\{\theta>0: K_{a} \leq K_{s}\right\}$, in accordance with [13].

\subsection{Martingale Bound for EDF Policy}

The EDF policy bases its behavior on the deadline associated with any service. In fact, the EDF scheduler serves the request that has the smallest leftover deadline. Consequently, the EDF scheduling for our case is given by:

$$
S^{i}=\left[S^{\text {Tot }}(m, n)-\sum_{j \neq i} A^{j}\left(m, \hat{m}+\left[d_{j}-d_{i}\right]\right)\right]_{+} \mathbf{1}_{\hat{n}>x},
$$

where $d_{j}, d_{i} \in\left\{\beta_{\mathcal{H}}, \beta_{\mathcal{G}}, d_{\mathcal{C}}, d_{\mathcal{D}}\right\}$, and the indicator function assumes value 1 when the condition $n-m>x$ is satisfied, and is zero otherwise.

By following the same procedure detailed in the FIFO policy case, and according to the standard literature [32], we can resort to the supermartingale process in which we define $M_{A^{i}}$, and $M_{S^{j}}$ as

$$
\begin{gathered}
M_{A^{i}} \approx h_{A^{i}}\left(a_{n}^{i}\right) e^{\theta\left(A^{i}(k, n)-(n-k) K^{A^{i}}\right)}, \\
M_{A^{-i}} \approx h_{A^{-i}}\left(a_{n}^{-i}\right) e^{\theta\left(A^{-i}(n-k+\min \{x, y\})-(n-k+\min \{x, y\}) K^{A^{-i}}\right)}, \\
M_{S^{j}} \approx h_{S^{j}}\left(s_{\tau_{j}}\right) e^{\theta\left(\tau_{j} K_{s}-S^{j}\left(\tau_{j}\right)\right)},
\end{gathered}
$$

where $y$ represents the sum of the difference of deadlines associated with the tagged flow and the other flows. According to literature, when $y \geq 0$, we have (26).

$$
\begin{aligned}
& \mathbb{P}\left(W^{1}(n) \geq k\right) \\
& \quad \leq \mathbb{P}\left(\sup _{0 \leq k \leq n}\left\{A^{1}(k, n)+\sum_{q \in\{\mathcal{H}, \mathcal{G}, \mathcal{C}, \mathcal{D}\} \backslash\{i\}} A^{q}(n-k) K^{A^{q}} A^{q}(n-k+y)-\sum_{j=2}^{3} S^{j}\left(\tau_{j}\right)\right\} \geq 0\right) .
\end{aligned}
$$

By distinguishing the following cases,

1. $n \geq k, n<k-y$;

2. $n \geq n-y$. 
In case (1) we have that $\mathbb{P}\left(W^{i}(n) \geq k\right)$ by (27),

$$
\mathbb{P}\left(\sup _{k \leq n \leq n}\left\{A^{i}(k, n)+\sum_{q \in\{\mathcal{H}, \mathcal{G}, \mathcal{C}, \mathcal{D}\} \backslash\{i\}} A^{q}(n-k) K^{A^{q}}+\sum_{j=2}^{3}\left(\tau_{j} K_{s}-S^{j}\left(\tau_{j}\right)\right)\right\} \geq k K_{s}\right),
$$

from which we have

$$
\mathbb{P}(W(n) \geq k) \leq e^{-\theta^{*} k K_{s}} B,
$$

where

$$
B=\frac{\mathbb{E}\left[M_{A^{i}}(0)\right] \mathbb{E}\left[M_{S^{2}}(0)\right] \mathbb{E}\left[M_{S^{3}}(0)\right]}{H} .
$$

Differently, in case (2), we have,

$$
\begin{array}{r}
\mathbb{P}\left(\operatorname { s u p } _ { k \leq n \leq n } \left\{A^{1}(k, n)+\sum_{q \in\{\mathcal{H}, \mathcal{G}, \mathcal{C}, \mathcal{D}\} \backslash\{i\}} A^{q}(n-k+y)-(n-k) K^{A^{i}}-\right.\right. \\
\left.\left.\sum_{q \in\{\mathcal{H}, \mathcal{G}, \mathcal{C}, \mathcal{D}\} \backslash\{i\}}(n-k+y) K^{A^{q}}+\sum_{i=2}^{3}\left(\tau_{i} K_{s}-S^{i}\left(\tau_{i}\right)\right)\right\} \geq k K_{s}-y K^{A^{1}}\right) .
\end{array}
$$

By exploiting the supermartingale processes,

$$
\begin{aligned}
& \mathbb{P}\left(W^{i}(n) \geq k\right) \\
& \quad \leq e^{-\theta^{*} k K_{s}-\sum_{q \in\{\mathcal{H}, \mathcal{G}, \mathcal{C}, \mathcal{D}\} \backslash\{i\}} A^{q}(n-k) K^{A^{q}} y K^{A^{q}}} B^{*},
\end{aligned}
$$

where

$$
B^{*}=\frac{\left[\prod_{i \in\{\mathcal{H}, \mathcal{G}, \mathcal{C}, \mathcal{D}\}} \mathbb{E}\left[M_{A^{i}}(0)\right]\right] \mathbb{E}\left[M_{S^{2}}(0)\right] \mathbb{E}\left[M_{S^{3}}(0)\right]}{H} .
$$

Due to the heterogeneity of the flows considered in terms of deadline constraints and also in reference to their access scheme, in order to derive the final e2e delay bound, we have to take into account different access delays for the different flows. For the traffic classes $\mathcal{H}$ and $\mathcal{G}$ the access contribution has been assumed to be deterministically equal to the computation request transmission time $\tau$ (in $m s$ ). Therefore, the reliability deadline target has to be scaled to $\tau \mathrm{ms}$, resulting in

$$
R=1-\mathbb{P}(W(u) \geq \hat{k}),
$$

where $\hat{k}=k-\tau$, and $k$ is the QoS constraint associated to the flow object of the analysis. Therefore, taking into account the access delay, the $B$ and $B^{*}$ terms have to be rewritten as

$$
B=\frac{\mathbb{E}\left[M_{A^{i}}(0)\right] \mathbb{E}\left[M_{S^{1}}(0)\right] \mathbb{E}\left[M_{S^{2}}(0)\right] \mathbb{E}\left[M_{S^{3}}(0)\right]}{H},
$$

and as (35), respectively,

$$
B^{*}=\frac{\left[\prod_{i \in\{\mathcal{H}, \mathcal{G}, \mathcal{C}, \mathcal{D}\}} \mathbb{E}\left[M_{A^{i}}(0)\right]\right] \mathbb{E}\left[M_{S^{2}}(0)\right] \mathbb{E}\left[M_{S^{1}}(0)\right] \mathbb{E}\left[M_{S^{2}}(0)\right] \mathbb{E}\left[M_{S^{3}}(0)\right]}{H},
$$

where $S^{1}$ is the service of the access subsystem [13].

\section{Performance Analysis}

This section presents the numerical simulation results which confirm the effectiveness of the proposed analysis in reference to the scenario presented in $[25,26]$ and here assumed. Therefore, the simulation parameters have been selected considering a square area network with a side of $20 \mathrm{~m}$, where the transmission power is the same for all the devices and it 
has been set equal to $1 \mathrm{~W}$. For the devices belonging to the communities $\mathcal{C}$ and $\mathcal{D}$, the QoS deadlines have been set equal to $k=35 \mathrm{~ms}$ and $k=40 \mathrm{~ms}$, respectively. However, for the flows $\mathcal{H}$ and $\mathcal{G}$, the corresponding QoS deadlines have been set as $k=25 \mathrm{~ms}$ and $k=30 \mathrm{~ms}$, respectively. The packets injected into the network have been modeled with a fixed size equal to 10 Mbits. Furthermore, between the tagged SBS and the linked devices and VEs, a channel bandwidth of $15 \mathrm{GHz}$ for both uplink and downlink communications has been considered. Then, the computation time for the computing subsystem has been modeled as hyperexponentially distributed with a mean service time of $5.25 \mathrm{~ms}$. In addition, it is important to highlight that the value of $\tau$ depends on the channel bandwidth [25-27].

Figure 4 exhibits the reliability of the system in providing services to the $\mathrm{uVR}$ user, as the bandwidth increases. As is noticeable from the figure, the theoretical bounds formulated (MG) for both the FIFO and the EDF scheduling policies are remarkably close to the actual simulation results (SR). Furthermore, Figure 4 shows performance improvement as the available bandwidth increases, which is an intuitive fact. Similarly, the curves confirm the validity of the EDF policy in comparison to a FIFO scheduling strategy, achieving higher reliability values by adopting the EDF in comparison to the FIFO alternative. The results have been obtained considering $v=4, p_{\mathcal{H}}=p_{\mathcal{G}}=0.01$, and $p_{c}=p_{d}=0.012$.

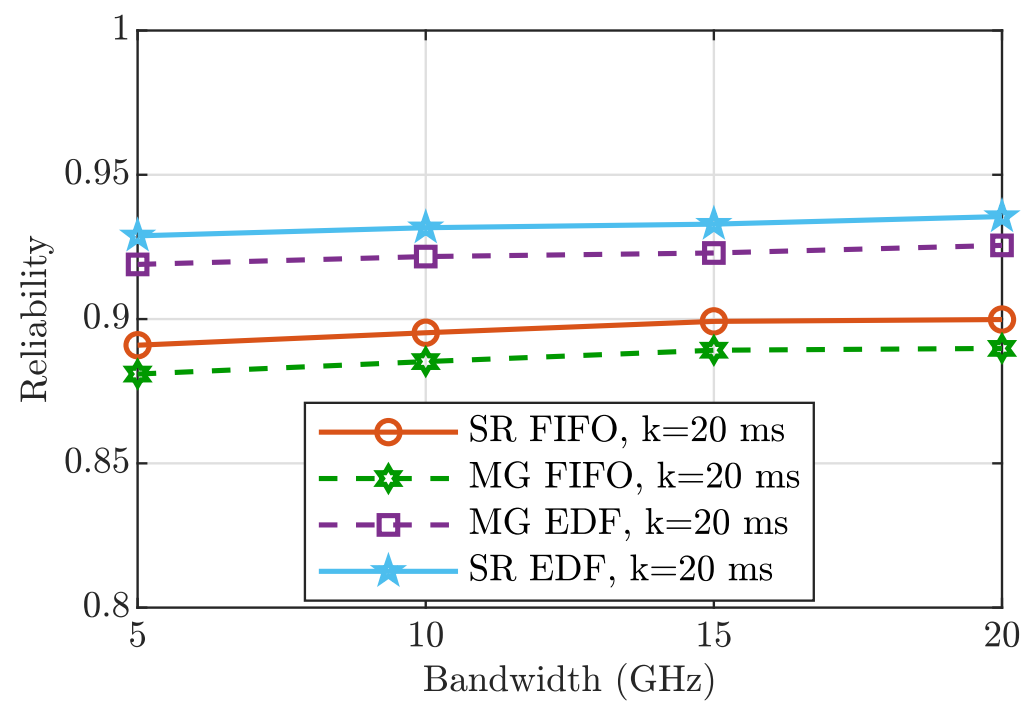

Figure 4. Reliability as a function of the bandwidth, considering $p_{\mathcal{H}}, p_{\mathcal{G}}=0.01$, and $p_{c}, p_{d}=0.012$.

An insight about the channel behavior is also provided in Figure 5, where the susceptibility of the THz channel to the molecular absorption coefficient is highlighted. In fact, as it is evident from the figure, such a coefficient considerably affects the system's reliability, resulting in remarkable performance degradation. In this case, the accuracies of the MG analytical predictions are also evident when compared with the SR curve, exhibiting the valuable behavior of the e2e delay analysis provided.

Figure 5 shows the results obtained considering again $v=4, p_{\mathcal{H}}=p_{\mathcal{G}}=0.01$, and $p_{c}=p_{d}=0.012$.

However, under the assumption of $p_{\mathcal{H}}=0.014, p_{\mathcal{G}}=0.01$, and $p_{c}, p_{d}=0.01$, Figure 6 depicts the trend of the reliability experienced by the flow $\mathcal{H}$ as the value of $v$ grows. Clearly, by increasing the number of devices belonging to $\mathcal{C}$ and $\mathcal{D}$, the traffic injected into the network grows, resulting in reliability degradation. In this case, both the MG curves are clearly close to the SR curves, confirming once again the goodness of the analysis formulated, exhibiting the superiority of the EDF approach to reach reliability values greater than those achieved under the FIFO assumption. The same reliability trend is confirmed by Figure 7 , in which $p_{\mathcal{H}}=0.014$, and $p_{\mathcal{G}}=0.01$ have been assumed, and the reliability is expressed as a function of $p_{c}$ and $p_{d}$, which are supposed equal to each other. 


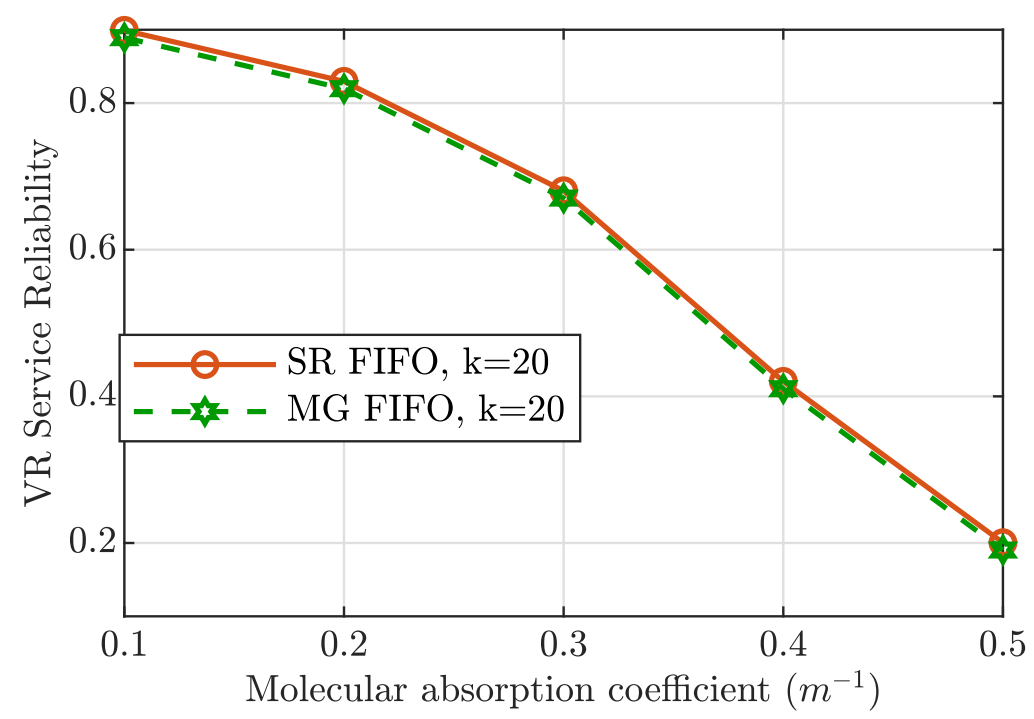

Figure 5. Reliability as a function of the molecular absorption, considering $p_{\mathcal{H}}, p_{\mathcal{G}}=0.01$, and $p_{c}, p_{d}=0.012$.

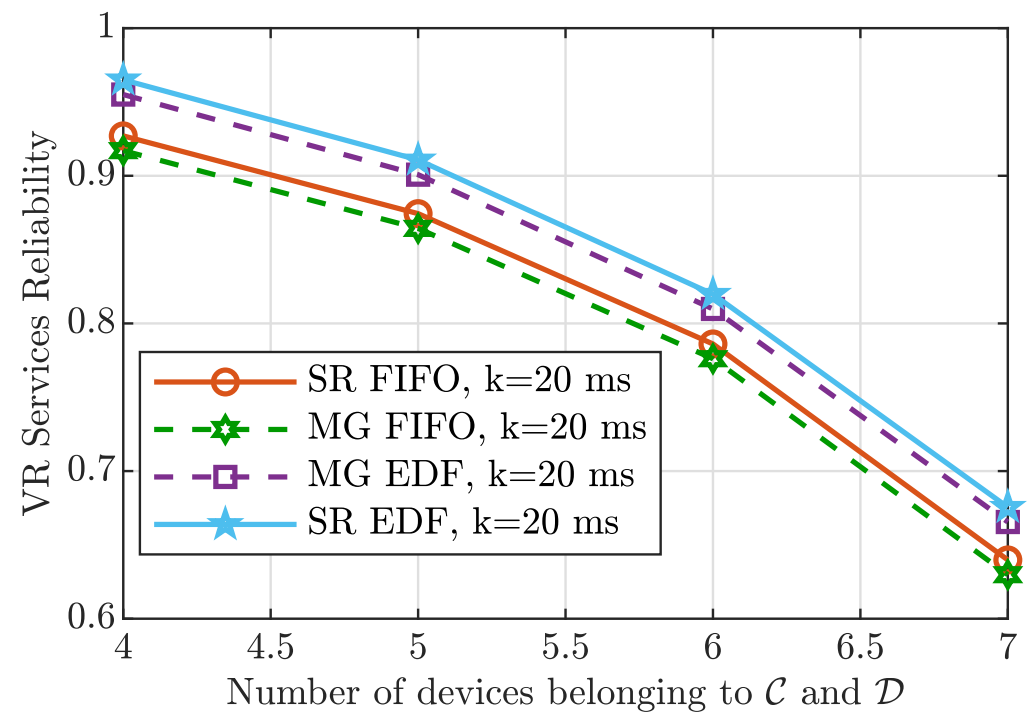

Figure 6. Reliability as a function of the number of devices belonging to $\mathcal{C}$ and $\mathcal{D}$, considering $p_{\mathcal{H}}=0.014, p_{\mathcal{G}}=0.01$, and $p_{c}, p_{d}=0.01$.

From Figure 7 , it follows that the reliability decreases when both $p_{c}$ and $p_{d}$ grow, and that the EDF is also the suitable strategy here. Likewise, the tightness between the MG and the SR curves is evident from Figure 7. Furthermore, Figure 8 shows the probabilistic bound of the reliability experienced by the flow stemmed by the device community $\mathcal{C}$. The results have been obtained supposing $p_{\mathcal{H}}=0.014$, and $p_{\mathcal{G}}=0.01$. Even in this case, the analytical MG curves achieve significantly accurate predictions about the actual system behavior (SR curves).

Then, Figure 9 confirms the same trend as $p_{\mathcal{H}}$ and $p_{\mathcal{G}}$ increase, supposing, also in this case, that $p_{\mathcal{H}}$ and $p_{\mathcal{G}}$ are equal to each other. In fact, the system reliability becomes worse as the value set for both $p_{\mathcal{H}}$ and $p_{\mathcal{G}}$ increases, achieving a better performance by applying the EDF scheduling strategy instead of the FIFO policy. The accuracy of the MG curves in fitting the SR behavior are also clear in this figure. 


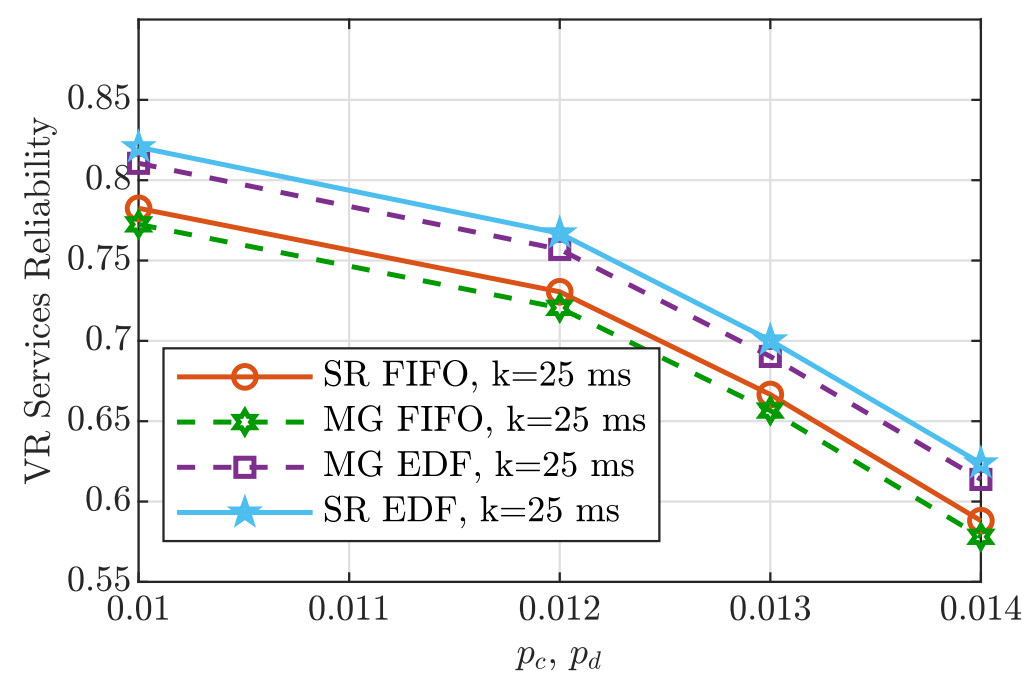

Figure 7. Reliability as a function of $p_{c}, p_{d}$, considering $p_{\mathcal{H}}=0.014$, and $p_{\mathcal{G}}=0.01$.

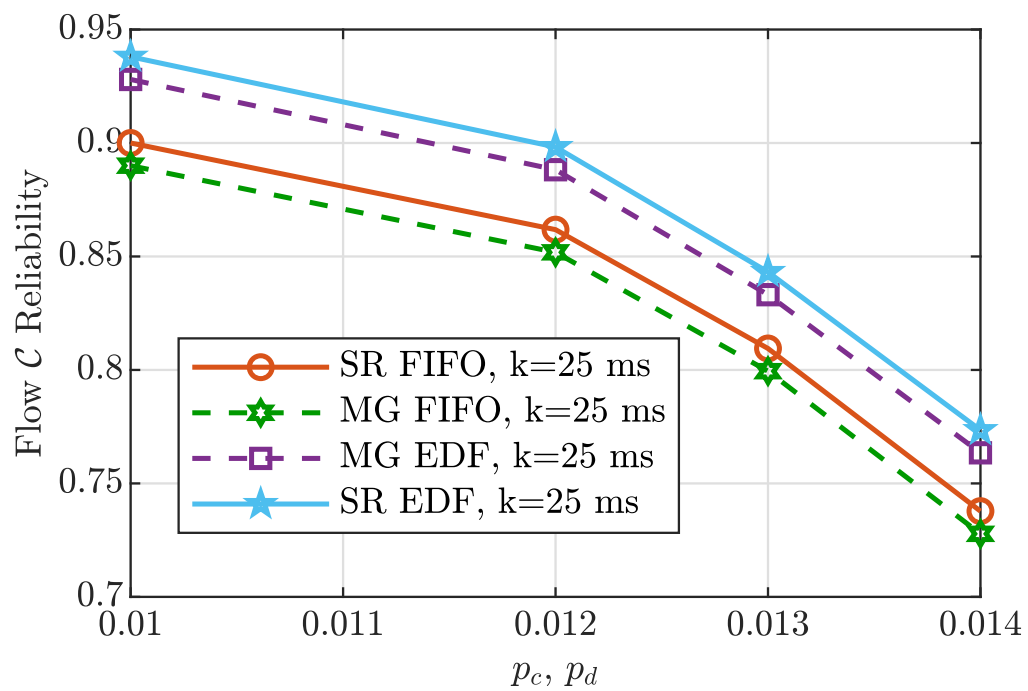

Figure 8. Reliability as a function of $p_{c}, p_{d}$, considering $p_{\mathcal{H}}=0.014$, and $p_{\mathcal{G}}=0.01$.

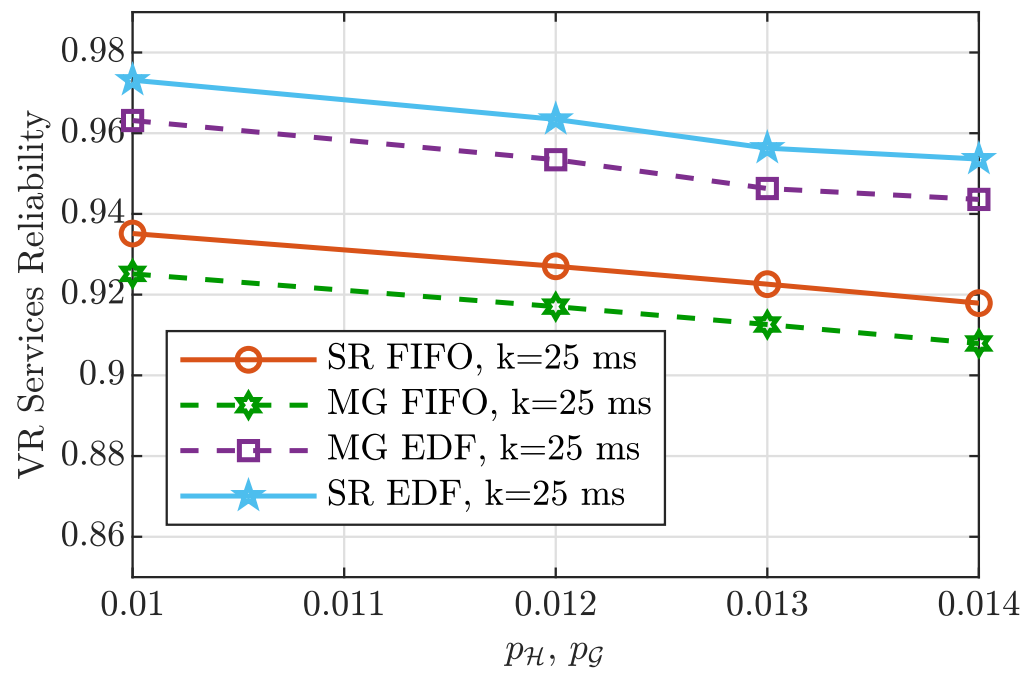

Figure 9. Reliability as a function of $p_{\mathcal{H}}, p_{\mathcal{G}}$, considering $p_{c}=0.01$, and $p_{d}=0.01$.

In conclusion, the results obtained exhibit the suitability of the formulated bound to provide performance analysis of $6 \mathrm{G} \mathrm{THz}$ network environments to support $\mathrm{uVR}$ services. 
In this sense, the framework formulated performs accurate analytical predictions of the system behavior of the object of study, for both the scheduling policies assumed. In fact, the accuracy of the analytical predictions provided results that were useful for actuating admission control policies during the network planning and design process, allowing us to properly forecast the system's behavior, providing information about the suitable network dimensioning (e.g., the channel bandwidth and the computing system load) in order to guarantee preset QoS target deadlines.

\section{Conclusions}

This paper has presented an in-depth per-flow e2e delay analysis, considering a number of concurrent traffic flows greater than two, under the assumption of different scheduling policies, within a 6G PEC network operating over THz frequencies. Moreover, by combining the SNC principles with the martingale envelopes theory, accurate performance bounds have been formulated for different scheduling schemes. The scheduling policies analyzed are FIFO and EDF. In both cases, the closeness of the proposed analytical predictions with the actual simulation results is evident. In fact, the numerical results clearly highlight the validity of the proposed stochastic analysis and its accuracy in predicting the actual behavior of the $6 \mathrm{G}$ network environment.

In reference to the future research directions, a very interesting topic needing deeper exploration may be represented by the impact of multi-user flows, heterogeneous in QoS requirements and stemming from both IoT devices and $\mathrm{uVR}$ users, on the arrivals waiting queue. In fact, the presence of multi-sources originates batch arrivals that cross the target traffic flow, giving rise to a non-negligible impact of the packets' sorting on the waiting time of the $\mathrm{uVR}$ flow due to the batch arrivals.

Funding: This research received no external funding.

Institutional Review Board Statement: Not applicable.

Informed Consent Statement: Not applicable.

Conflicts of Interest: The author declares no conflict of interest.

\section{Appendix A}

Hereafter, we present some fundamental martingale theory definitions $[13,28,33]$.

Definition A1. Submartingale Process: Let $\left\{\mathcal{F}_{n}\right\}_{n}$ be a filtration such that the stochastic process $\left\{Y_{n}\right\}_{n}$ is $\mathcal{F}_{n^{-}}$measurable. $\left\{Y_{n}\right\}_{n}$ is a submartingale process if, for any time $n \geq 1$, $Y_{1}, Y_{2}, \ldots$ it satisfies

$$
\begin{gathered}
E\left[\left|Y_{n}\right|\right]<\infty, \\
E\left[Y_{n+1} \mid \mathcal{F}_{n}\right] \geq Y_{n} .
\end{gathered}
$$

Definition A2. Martingale Process: The stochastic process $Y_{1}, Y_{2}, \ldots$ is a martingale process if, for any time $n \geq 1$, it satisfies

$$
\begin{gathered}
E\left[\left|Y_{n}\right|\right]<\infty, \\
E\left[Y_{n+1} \mid \mathcal{F}_{n}\right]=Y_{n} .
\end{gathered}
$$

Definition A3. Supermartingale Process: The stochastic process $Y_{1}, Y_{2}, \ldots$ is a supermartingale process if, for any time $n \geq 1$, it satisfies

$$
\begin{gathered}
E\left[\left|Y_{n}\right|\right]<\infty, \\
E\left[Y_{n+1} \mid \mathcal{F}_{n}\right] \leq Y_{n} .
\end{gathered}
$$


Definition A4. Arrival Martingale: The arrival process $A$ exhibits martingale arrivals if, for any $\theta>0, \exists K_{a} \geq 0$, and $h_{a}: C(X) \rightarrow \mathbb{R}^{+}$it satisfies

$$
h_{a}\left(X_{b}\right) e^{\theta\left(A(b)-b K_{a}\right)}, b \geq 1 .
$$

and the process is supermartingale.

Definition A5. Service Martingales: The service process $S$ exhibits martingale arrivals if, for any $\theta>0, \exists K_{s} \geq 0$, and $h_{s}: C(X) \rightarrow \mathbb{R}^{+}$that satisfies

$$
h_{S}\left(S_{b}\right) e^{\theta\left(b K_{2}-S(b)\right)}, b \geq 1,
$$

and the process is supermartingale.

Definition A6. Arrivals/Service Martingales: Let $R_{1}, R_{2}, \ldots$, be i.i.d random variables, in which the corresponding distributions are non-negative. By assuming generically $A(b)=$ $S(b)=\sum_{g=1}^{b} R_{g}$, it follows that both $A$ and $S$ admit arrival and service martingales, respectively.

For a detailed exposition we refer to [13]. Furthermore, in accordance with [13], in Definition A5, the number of packets served at time $b$ is represented by $S_{b}$, while in Definitions A4 and A5, C is the range operator.

\section{References}

1. Kasgari, A.T.Z.; Saad, W.; Debbah, M. Human-in-the-Loop Wireless Communications: Machine Learning and Brain-Aware Resource Management. CoRR 2018, 67, 7727-7743.

2. Saad, W.; Bennis, M.; Chen, M. A Vision of 6G Wireless Systems: Applications, Trends, Technologies, and Open Research Problems. IEEE Netw. 2020, 34, 134-142. [CrossRef]

3. Chen, M.; Saad, W.; Yin, C. Virtual Reality over Wireless Networks: Quality-of-Service Model and Learning-Based Resource Management. arXiv 2018, arXiv:1703.04209.

4. Al-Eryani, Y.F.; Hossain, E. Delta-OMA (D-OMA): A New Method for Massive Multiple Access in 6G. arXiv 2019, arXiv:1901.07100.

5. Gomes de Sa, A.; Zachmann, G. Virtual reality as a tool for verification of assembly and maintenance processes. Comput. Graph. 1999, 23, 389-403. [CrossRef]

6. Lin, P.; Song, Q.; Wang, D.; Yu, R.; Guo, L.; Leung, V. Resource Management for Pervasive Edge Computing-Assisted Wireless VR Streaming in Industrial Internet of Things. IEEE Trans. Ind. Inform. 2021. [CrossRef]

7. Liu, Y.; Liu, J.; Argyriou, A.; Ci, S. MEC-Assisted Panoramic VR Video Streaming Over Millimeter Wave Mobile Networks. IEEE Trans. Multimed. 2019, 21, 1302-1316. [CrossRef]

8. Bastug, E.; Bennis, M.; Medard, M.; Debbah, M. Toward Interconnected Virtual Reality: Opportunities, Challenges, and Enablers. IEEE Commun. Mag. 2017, 55, 110-117. [CrossRef]

9. Fidler, M.; Rizk, A. A Guide to the Stochastic Network Calculus. IEEE Commun. Surv. Tutor. 2015, 17, 92-105. [CrossRef]

10. Jiang, Y. A Basic Stochastic Network Calculus. SIGCOMM Comput. Commun. Rev. 2006, 36, 123-134. [CrossRef]

11. Li, C.; Burchard, A.; Liebeherr, J. A Network Calculus With Effective Bandwidth. IEEE/ACM Trans. Netw. 2007, 15, 1442-1453. [CrossRef]

12. Fidler, M. Survey of deterministic and stochastic service curve models in the network calculus. IEEE Commun. Surv. Tutor. 2010, 12, 59-86. [CrossRef]

13. Poloczek, F.; Ciucu, F. Service-martingales: Theory and applications to the delay analysis of random access protocols. In Proceedings of the 2015 IEEE Conference on Computer Communications (INFOCOM), Hong Kong, China, 26 April-1 May 2015; pp. 945-953. [CrossRef]

14. Park, J.; Bennis, M. URLLC-eMBB Slicing to Support VR Multimodal Perceptions over Wireless Cellular Systems. In Proceedings of the 2018 IEEE Global Communications Conference (GLOBECOM), Abu Dhabi, United Arab Emirates, 9-13 December 2018; pp. 1-7. [CrossRef]

15. Sun, Y.; Chen, Z.; Tao, M.; Liu, H. Communication, Computing and Caching for Mobile VR Delivery: Modeling and Trade-Off. In Proceedings of the 2018 IEEE International Conference on Communications (ICC), Kansas City, MO, USA, 20-24 May 2018; pp. 1-6.

16. Popovski, P.; Stefanović, Č.; Nielsen, J.J.; de Carvalho, E.; Angjelichinoski, M.; Trillingsgaard, K.F.; Bana, A.S. Wireless Access in Ultra-Reliable Low-Latency Communication (URLLC). IEEE Trans. Commun. 2019, 67, 5783-5801. [CrossRef]

17. Angjelichinoski, M.; Trillingsgaard, K.F.; Popovski, P. A Statistical Learning Approach to Ultra-Reliable Low Latency Communication. IEEE Trans. Commun. 2019, 67, 5153-5166. [CrossRef] 
18. Li, Y.; Luo, X.; Lobo, E.; Pilco, A.; Chen, Y. Wireless Ad Hoc Network Simulation Based on Virtual Reality Technology. In Proceedings of the 2017 International Conference on Virtual Reality and Visualization (ICVRV), Zhengzhou, China, 21-22 October 2017; pp. 458-460.

19. Ahn, J.; Kim, Y.Y.; Kim, R.Y. Delay oriented VR mode WLAN for efficient Wireless multi-user Virtual Reality device. In Proceedings of the 2017 IEEE International Conference on Consumer Electronics (ICCE), Las Vegas, NV, USA, 8-10 January 2017; pp. 122-123.

20. Hu, Y.; Li, H.; Chang, Z.; Han, Z. End-to-End Backlog and Delay Bound Analysis for Multi-Hop Vehicular Ad Hoc Networks. IEEE Trans. Wirel. Commun. 2017, 16, 6808-6821. [CrossRef]

21. Hu, Y.; Li, H.; Han, Z. Delay Bound Analysis Using Martingale for Multimedia DTN under Heterogeneous Network for High-Speed Trains. In Proceedings of the 2015 IEEE Global Communications Conference (GLOBECOM), San Diego, CA, USA, 6-10 December 2015; pp. 1-6. [CrossRef]

22. Qi, R.; Chi, X.; Zhao, L.; Yang, W. Martingales-Based ALOHA-Type Grant-Free Access Algorithms for Multi-Channel Networks With mMTC/URLLC Terminals Co-Existence. IEEE Access 2020, 8, 37608-37620. [CrossRef]

23. Shortle, J.; Thompson, J.; Gross, D.; Harris, C. Fundamentals of Queueing Theory; Wiley Series in Probability and Statistics; John Wiley \& Sons, Incorporated: Hoboken, NJ, USA, 2018.

24. Mutairi, A.; Roy, S.; Hwang, G. Delay Analysis of OFDMA-Aloha. IEEE Trans. Wirel. Commun. 2013, 12, 89-99. [CrossRef]

25. Jain, I.; Kumar, R.; Panwar, S. The Impact of Mobile Blockers on Millimeter Wave Cellular Systems. IEEE J. Sel. Areas Commun. 2019, 37, 854-868. [CrossRef]

26. Petrov, V.; Moltchanov, D.; Koucheryavy, Y. Interference and SINR in Dense Terahertz Networks. In Proceedings of the 2015 IEEE 82nd Vehicular Technology Conference (VTC2015-Fall), Boston, MA, USA, 6-9 September 2015; pp. 1-5.

27. Wang, C.C.; Yao, X.W.; Han, C.; Wang, W.L. Interference and Coverage Analysis for Terahertz Band Communication in Nanonetworks. In Proceedings of the GLOBECOM 2017-2017 IEEE Global Communications Conference, Singapore, 4-8 December 2017; pp. 1-6. [CrossRef]

28. Liu, T.; Li, J.; Shut, F.; Han, Z. Quality-of-Service Driven Resource Allocation Based on Martingale Theory. In Proceedings of the 2018 IEEE Global Communications Conference (GLOBECOM), Abu Dhabi, United Arab Emirates, 9-13 December 2018; pp. 1-6. [CrossRef]

29. Fidler, M. An End-to-End Probabilistic Network Calculus with Moment Generating Functions. In Proceedings of the 2006 14th IEEE International Workshop on Quality of Service, New Haven, CT, USA, 19-21 June 2006; pp. 261-270.

30. Williams, D. Probability with Martingales; Cambridge University Press: Cambridge, UK, 1991. [CrossRef]

31. Ethier, S.; Kurtz, T. Markov Processes: Characterization and Convergence; Wiley Series in Probability and Statistics; Wiley: Hoboken, NJ, USA, 2009.

32. Ciucu, F. Exponential supermartingales for evaluating end-to-end backlog bounds. SIGMETRICS Perform. Eval. Rev. 2007, 35, 21-23. [CrossRef]

33. Fantacci, R.; Picano, B. End-to-End Delay Bound for Wireless uVR Services over 6G Terahertz Communications. IEEE Internet Things J. 2021. [CrossRef] 